\title{
Recovery of the Irreversible Crystallinity of \\ Nanocellulose by Crystallite Fusion: A Strategy for Achieving Efficient Energy Transfers in Sustainable Biopolymer Skeletons
}

Kazuho Daicho ( $\nabla$ daichok@g.ecc.u-tokyo.ac.jp )

The University of Tokyo https://orcid.org/0000-0003-4203-2257

Kayoko Kobayashi

Kyoto University

Shuji Fujisawa

The University of Tokyo

Tsuguyuki Saito

The University of Tokyo

Physical Sciences - Article

Keywords: cellulose nanofibers, crystallinity, grain boundary, fusion, chitin

Posted Date: July 28th, 2021

DOI: https://doi.org/10.21203/rs.3.rs-518217/v4

License: (c) (i) This work is licensed under a Creative Commons Attribution 4.0 International License.

Read Full License

Version of Record: A version of this preprint was published at Angewandte Chemie International Edition on October 7th, 2021. See the published version at https://doi.org/10.1002/anie.202110032. 


\section{Abstract}

Crystallite refers to a single crystalline grain in crystal aggregates, and multiple crystallites form a grain boundary or the inter-crystallite interface. A grain boundary is a structural defect that hinders the efficient directional transfer of mechanical stress or thermal phonons in crystal aggregates. We observed that grain boundaries within an aggregate of a-few-nanometers-wide fibrillar crystallites of wood cellulose were crystallized by enhancing their inter-crystallite interactions; multiple crystallites were coupled into single fusion crystals without passing through a melting or dissolving state. Accordingly, the crystallinity of wood cellulose, which has been considered irreversible once decreased, was significantly enhanced, and the thermal energy transfer in the aggregate was improved. Other fibrillar crystallites of crab shell chitin also showed a similar fusion phenomenon by enhancing the inter-crystallite interactions. These findings imply that such crystallite fusion naturally occurs in biological structures with network skeletons of aggregated fibrillar crystallites.

\section{Introduction}

Mechanical and thermal energy transfers in crystal aggregates are governed by a grain boundary or the interface where multiple crystallites meet. ${ }^{[1]}$ Proper crystallite interactions at the grain boundary can allow mechanical stress or thermal phonons to directionally transfer in the aggregate; however, a grain boundary is often a structural defect that deforms under stress or scatters phonons. ${ }^{[2-3]}$ This feature becomes pronounced when the crystallites have nanoscale dimensions and form a large area of the grain boundary. In the field of nanotechnology, it is currently a challenge to exploit the potential of such nanoscale crystallites, including biopolymer fibrils and clay platelets, in bulk aggregates or composites by tailoring the interactions between crystallites or with other components. ${ }^{[4-6]}$

Ideal energy transfer in crystal aggregates must be realized by crystallization of the grain boundary. If multiple crystallites can be coupled into single fusion crystals by forming a bulk aggregate from their dispersions, scalable polycrystalline materials with more efficient mechanical and thermal energy transfers will be produced. However, crystallites often have disordered structures or defects at their surfaces, ${ }^{[4,7]}$ hindering efficient energy transfer.

Herein, we report that grain boundaries within an aggregate of a-few-nanometers-wide crystalline cellulose nanofibers (CNFs) were crystallized by enhancing the inter-CNF interactions. CNFs are sustainable materials with excellent mechanical and thermal properties, which are produced as a water dispersion using wood pulps as the raw material. ${ }^{[8]}$ Their elemental unit is a crystallite consisting of uniaxially oriented molecular chains, known as a cellulose microfibril. ${ }^{[9-10]}$ In the dispersion, the surface molecules of CNFs have a thermodynamically stable, non-crystalline conformation and are uniaxially oriented, similar to the inner crystalline molecules. ${ }^{[10-12]}$

We previously showed that the crystallinity of CNFs significantly decreased when aggregated microfibrils in a pulp dispersed as CNFs or separated into individual crystallites. ${ }^{[11,13]}$ This phenomenon was 
interpreted to result from the exposure of the grain boundary that was partially crystallized in the microfibril aggregates. This study was aimed at recovering the decreased crystallinity of the CNFs by assembly. We also verified if the crystallization of the grain boundary led to greater efficiency in the thermal energy transfer of bulk crystal aggregates.

\section{Results And Discussion}

Aggregating CNFs. Scheme 1 shows the procedure for enhancing the inter-CNF interactions in a bulk aggregate. The CNF dispersion was prepared by wet disintegration of a TEMPO-oxidized wood pulp. ${ }^{[14]}$ In the TEMPO-oxidation reaction, the $\mathrm{C} 6$ hydroxy groups exposed on the microfibril surfaces are regioselectively oxidized, such that the resulting CNFs possess a high surface density of carboxy groups (up to 1.7 groups per square nanometer). The carboxy groups are $\mathrm{Na}^{+}$type at the initial step. By the dissociation of the carboxy groups, the CNFs are stably dispersed in water and spontaneously form a nematic liquid-crystalline phase where the CNFs are uniaxially oriented in places (Scheme 1a). ${ }^{[15]}$ In this study, the nematic-ordered CNF dispersion was assembled into dry sheets by two processes. One process was freeze drying from a $30 \%$ tert-butyl alcohol-containing diluted dispersion, followed by pressing of the dried aerogel-like product (sample $i) .{ }^{[16]}$ The other process was evaporative drying via solvent casting of the CNF dispersion at $40{ }^{\circ} \mathrm{C}$ under a high relative humidity of $80 \%$, resulting in the formation of transparent and flat dry sheets (sample ii). ${ }^{[17]}$ Sample ii was further processed with a dilute acid solution to convert the carboxy groups from sodium salt into protonated acid form, $\mathrm{H}$ type (sample iii, see Figure S1a for the evidence of protonation) ${ }^{[18]}$ followed by a hydrothermal treatment using a common autoclave at $135^{\circ} \mathrm{C}$ and $212 \mathrm{kPa}$ (sample $\mathrm{iv}$ ). The hydrothermal treatment was adopted to enable the CNFs to rearrange and stabilize their interaction. Note that the CNFs remained solid, without melting or dissolving, under the conditions adopted in this study.

The assembled state of the CNFs in each sheet is illustrated in Scheme 1b: roughly oriented in sample $i$, and highly oriented in samples $i i-i v$. Meanwhile, the CNFs of samples $i-i v$ were on average packed parallel to the sheet surface (see Figures S2 for X-ray diffraction (XRD) diagrams). ${ }^{[17]}$ The CNFs in samples iii and $i v$ were also hydrogen-bonded with one another via the surface carboxy groups, as analyzed by Fourier-transform infrared (FTIR) spectroscopy (Figure S1a), and those in sample iv were more strongly hydrogen-bonded (Figure $S 1 b, c)$. Accordingly, the porosity of the samples decreased in the order of sample $i>i i>i i i>i v$, at $16 \%, 10 \%, 9 \%$, and $4 \%$, respectively. The specific surface area (SSA) of sample $i$ was $\sim 6.3 \mathrm{~m}^{2} \mathrm{~g}^{-1}$, as measured by nitrogen adsorption analysis (Figure S3), whereas those of samples ii, iii, and iv were all below the limit of detection $\left(\sim 2 \mathrm{~m}^{2} \mathrm{~g}^{-1}\right)$. Therefore, the CNFs in each sample were considered to interact more closely or strongly in the order of sample $i<i i<i i i<i v$.

Recovery of Crystallinity. Figure 1a shows powder XRD profiles of the oxidized starting pulp and CNF samples $i-i v$. The XRD profiles of the pulp and sample $i$ notably differed; all the peaks broadened and their intensities decreased. From sample $i$ to $i$, the peak widths narrowed. From sample $i i i$ to $i v$, the peaks 
further sharpened and intensified, and the $\left(\begin{array}{ll}2 & 0\end{array}\right)$ peak shifted slightly to the high-angle side. Finally, sample iv exhibited a distinct XRD profile rather than the starting pulp.

The nuclear magnetic resonance (NMR) spectra for the samples are shown in Figure 1b. In the C4 and C6 regions of the NMR spectra, the crystalline signals centered at $88 \mathrm{ppm}$ (C4) and $65 \mathrm{ppm}$ (C6) remarkably decreased at the first step of the pulp-sample $i$ conversion and increased in the order of samples ii, iii, and $i v$. Meanwhile, the non-crystalline signals at $84 \mathrm{ppm}$ (C4) and $62 \mathrm{ppm}$ (C6) increased at the first step and then decreased in the order of samples ii, iii, and $i v$.

As measures of the crystallinity, the Scherrer's crystal sizes of the $(200)$ plane and crystallinity indices were calculated from the XRD profiles and NMR spectra, respectively (Figure 1c,d). ${ }^{[11]}$ The crystallinity indices were expressed in two different forms as area ratios of the crystalline and non-crystalline signals in the $\mathrm{C} 4$ and $\mathrm{C} 6$ regions. Note that the crystalline $\mathrm{C} 6$ signal arises from the $\mathrm{C} 6$ carbon atoms turning the C6- 06 bond to the "trans-gauche $(t g)$ " configuration against the $\mathrm{C} 5-05 / \mathrm{C} 4-\mathrm{C} 5$ bonds in anhydroglucose units. ${ }^{[10]}$ Moreover, the crystalline $\mathrm{C} 4$ conformation is fixed with the intra/inter-molecular hydrogen bonds via the tg-configurational $\mathrm{C} 6$ hydroxy groups. The crystallinity indices thus reflect the degree of intra-/inter-molecular hydrogen bonding.

The $(200)$ crystal size decreased from $3.5 \mathrm{~nm}$ to $2.0 \mathrm{~nm}$ by the pulp-sample $i$ conversion, and increased up to $3.8 \mathrm{~nm}$ in the order of samples ii, iii, and iv (Figure 1c). This increase in the crystal size explained the slight shift of the (2 00 ) peak position to the high-angle side, according to a previous report. ${ }^{[19]}$ Also, the two crystallinity indices of the C4 and C6 carbon atoms decreased by $~ 30 \%$ from $40-50 \%$ on the conversion to sample $i$, and then increased by $\sim 30 \%$ for samples ii, iii, and iv (Figure 1d). For both the crystal size and crystallinity index, the initial decrease was interpreted to result from the increase in SSA by disintegration of the pulp into the CNF dispersion (see Introduction). ${ }^{[11]}$ The successive increase was thus attributed to assembly of the CNFs in dry sheets; the inter-CNF interaction was dominant in the recovery of crystallinity.

Interestingly, the final values for sample iv reached or even surpassed the crystal size and crystallinity indices of the starting pulp; the crystallinity of the CNFs was reversible (see Figure S4 for the repeatability data). The recovery of crystallinity was also demonstrated for other type of CNFs produced from chemically unmodified, raw pulps solely by wet disintegration (Figure S5). Furthermore, other fibrillar crystallites of a-chitin showed the recovery of crystallinity through the same process as the dispersion and assembly of CNFs in this study (Figure S6).

Inter-CNF interactions. The contribution of the inter-CNF interactions to the recovery of crystallinity was investigated in more detail using two additional surface-modified CNFs (Figure 2a): hydrophobic CNFs bearing bulky tetra-n-butylammonium (TBA) as the counter ion of the surface carboxy group; ${ }^{[20]}$ and polymer-covered CNFs adsorbing an amorphous, hydroxyethyl cellulose by $\sim 10 \% \mathrm{w} / \mathrm{w}^{[21]}$ The adsorption of $10 \%$ roughly corresponds to two HEC molecules per single CNF. These two CNF dispersions were 
assembled into transparent dry sheets through the same drying process as adopted in the preparation of samples ii and iii.

The TBA-bearing CNFs exhibited a significant recovery of the (2 00 ) crystal size (Figure 2b), whereas their crystallinity index stayed as low as $14 \%$ (Figure $2 \mathrm{c}$ ). These results provided a deeper understanding of the recovery of crystallinity. The TBA ions are coupled with carboxy groups localized on the hydrophilic (1 1 $0)$ and $\left(\begin{array}{lll}1 & -1 & 0\end{array}\right)$ surfaces (see inset in Figure 1a), such that the hydrophobic $(200)$ surface is exposed. Thus, the recovery of the crystal size indicated that TBA-bearing CNFs were stacked on the exposed (2 0 0 ) plane. Meanwhile, the low crystallinity index indicated that the inter-CNF hydrogen bonds between the hydrophilic surfaces were inhibited by the bulky TBA ions.

The HEC-covered CNFs showed different behaviors, in that both the crystal size and crystallinity index slightly increased (Figure 2b,c). HEC is amphiphilic, such that the entire CNF surfaces are sparsely covered with HEC at a CNF/HEC ratio of 9:1. ${ }^{[21]}$ The results in Figure 2b,c for the HEC-covered CNFs were thus attributed to "partially-blocked" inter-CNF interactions.

These results supported that the inter-CNF interactions were dominant in the recovery of crystallinity, indicating that the inter-CNF (2 00 ) stacking and hydrogen bonding contributed mainly to the recovery of the crystal size and crystallinity index, respectively, which was also supported by the results for samples $i i$ and $i i i$ in Figure $1 \mathrm{c}$,d. From the $\mathrm{Na}^{+}$-type sample $i$ i to the hydrogen-bonded $\mathrm{H}$-type sample $i i i$, the $\left(\begin{array}{lll}2 & 0 & 0\end{array}\right)$ crystal size was approximately constant, whereas the crystallinity index significantly increased.

The "hornification" phenomenon is well known in the field of wood and paper sciences. It implies that the crystallinity of cellulosic samples including CNFs slightly increases by the repeating cycle of wet-dry states. ${ }^{[22-23]}$ This phenomenon has been ambiguously interpreted to result from enhancement of the degree of hydrogen bonding in samples by drying, and its mechanism remains unclear. The results in this study suggested that hornification is based on inter-microfibril $\left(\begin{array}{lll}2 & 0 & 0\end{array}\right)$ stacking and hydrogen bonding.

Simulation. To assess the major configuration of CNFs in the interactions, an XRD profile of sample iv was compared with simulated profiles of possible inter-CNF configurations (Figures 3 and S7). The experimental profile was obtained by azimuthally integrating an XRD diagram of a sample iv sheet set parallel to the beam. We assumed no specific orientation of the crystal planes to the sheet surface by considering: 1) the similarity of the experimental profile in Figure 3 with that obtained by the reflection method for sample iv in Figure 1a, and 2) the twisting structure of the CNFs around the crystallographic $c$ axis. ${ }^{[24-26]}$

In the simulation, the structure of the single CNFs was assumed to be composed of 18 cellulose chains with a stacking mode of $2 / 3 / 4 / 4 / 3 / 2$, based on previous reports on the morphological analyses of single CNFs. ${ }^{[1,27]}$ This 18-chain model has flat two-molecule-wide $\left(\begin{array}{ll}2 & 0\end{array}\right)$ surfaces, enabling the CNFs to stably stack on the (2 00 ) plane (see the results for TBA-bearing CNFs in Figure 2). As shown in Figure 3, the CNFs assembled parallel to one another along the $c$ axis. Simulations of antiparallel assembly are shown 
in Figure S7. Coupling of only two CNFs was simulated here for simplicity but the reality should be more complex.

The highest $R^{2}$ value (0.897) was obtained for configuration \#12, where the modelled CNFs coupled facing their $\left(\begin{array}{lll}1 & 1 & 0\end{array}\right)$ surfaces. ${ }^{[28]}$ This configuration allows the CNFs to form inter-CNF hydrogen bonds, explaining the recovery of the crystal size and crystallinity index for sample $i v$. In addition, the (1 110$)$ plane has a larger surface free energy than the other planes. ${ }^{[29]}$ Thus, the CNF coupling for configuration \#12 reduces the free energy in the system to a greater extent and is the most stable of the possible interCNF configurations. The XRD profile of the starting pulp (Figure 1a) also showed the best fit $\left(R^{2}=0.883\right.$ ) with configuration \#12. A similar result was obtained for the antiparallel assembly shown in Figure S5; the best fit $\left(R^{2}=0.875\right)$ was achieved at a configuration that significantly reduced the $\left(\begin{array}{lll}1 & 1 & 0\end{array}\right)$ surface. In the antiparallel assembly, there existed no configuration where the CNFs coupled by facing the same crystal plane.

Configuration \#7 matched the experimental profile in terms of peak shape, especially at lower angles of $\sim 15^{\circ}$. However, its coefficient of correlation, $R^{2}(0.826)$, was the lowest (see Methods for the $R^{2}$

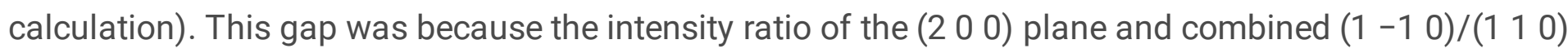
peaks in the simulation was significantly different from the corresponding ratio for the experimental profile. On closer inspection, the position of the combined $\left(\begin{array}{lll}1 & -1 & 0\end{array}\right) /\left(\begin{array}{ll}1 & 1\end{array}\right)$ peak in the simulation was also shifted by $\sim 1^{\circ}$ from the experimental peak.

Modelling. The recovery of the (20 0$)$ crystal size was easily interpreted as the phase extension of the (2 00 ) plane by CNF assembly. However, the mechanism for the recovery of crystallinity index must be considered. In Figure 1d, the C4- and C6-derived crystallinity indices were coordinated and recovered from the lowest degree of $15 \%$ (sample $i$ ) by up to $30 \%$ (sample iv). Considering that most of the C6 hydroxy groups exposed on the CNF surface were converted to carboxy groups, the recovery of crystallinity index was interpreted to result from conformational changes of not only the residual surface C6 hydroxy group but also the interior of each CNF structure via inter-CNF hydrogen bonding.

Figure 4 illustrates a model for the conformational changes induced by the inter-CNF hydrogen bonding. Three cases were assumed based on the hydrogen-bonding mode in the cellulose I type structure. ${ }^{[10]}$ In case 1 , the residual surface $\mathrm{C} 6$ hydroxy groups take the $\mathrm{tg}$ conformation from other noncrystalline states (gt or gg, see the section "Recovery of Crystallinity"). This change was the most plausible, but the maximum contribution to the recovery of crystallinity index was estimated to be $\sim 10 \%$, which was insufficient for explaining the recovery of $30 \%$. Cases 2 and 3 describe the conformational changes occurring at the interior of each CNF structure. In case 2, the $\mathrm{C} 6$ hydroxy groups in the surface molecules of each CNF facing inside adopt the $\operatorname{tg}$ conformation. In case 3 , the $\mathrm{C} 6$ hydroxy groups in the interior molecules facing the surface molecules adopt the $t g$ conformation. These two cases were assumed to result from some restraint imposed on the surface molecules via the inter-CNF hydrogen bonding. 
Bulk properties. The recovery of crystallinity was expected to improve the bulk properties of the CNF structures. Figure 5 shows the thermal diffusivity, $a$, and conductivity, $k$, of samples $i-i v$ as a function of the crystallinity index. With the recovery of crystallinity, both the diffusivity and conductivity significantly improved (see Figure 88 for the specific values of $a$ and $k$ divided by their bulk densities, showing the same trend). This trend is reasonable because heat transfers in a solid via phonon propagation. The phonon is an elastic wave and is often scattered at the grain boundary in a particle assembly. The recovery of crystallinity was caused by the inter-CNF interactions, which reduced the grain boundary and facilitated phonon propagation.

\section{Conclusions}

Enhancing the interaction between CNFs or a-few-nanometer-wide fibrillar crystallites of wood cellulose resulted in the coupling of multiple crystallites into single fusion crystals in a bulk aggregate without passing through a melting or dissolving state. The interaction was enhanced by the following steps: starting from a nematic liquid-crystalline dispersion where the CNFs were uniaxially oriented in places, forming a dense aggregate by condensation, and enabling the CNFs to bind with one another via hydrogen bonds at the grain boundary. These steps induced the conformational change of the constituent carbon atoms of the CNFs to be crystalline, and the phase extension of the crystal planes occurred. Accordingly, the lowered crystallinity of the CNFs, which was previously considered irreversible from an energetic point of view, was recovered, and thermal energy transfer in the aggregate was significantly improved.

CNFs have recently been produced in industrial settings, e.g. 1000 ton capacity in Japan in 2020, ${ }^{[30]}$ and this finding will contribute to the building of the technical bases for exploiting the potential of CNFs in bulk materials. This finding further offers a deeper understanding of "hornification", which has been ambiguously interpreted in the field of wood and paper sciences. Other fibrillar crystallites of crab shell chitin also showed a similar fusion phenomenon through the enhancement of their inter-crystallite interactions in the solid state. The implication is that such crystallite fusion naturally occurs in biological structures with network skeletons of aggregated fibrillar crystallites.

\section{Declarations}

Acknowledgements

A This research was partially supported by the JST-Mirai R\&D Program (JPMJMI17ED), JSPS Grant-inAids for Scientific Research (18K14501; 20J12793; 20K15567; 21H04733), a PHOENIX Grant-in-Aid, and the Research Program for Next Generation Young Scientists of "Network Joint Research Center for Materials and Devices: Dynamic Alliance for Open Innovation Bridging Human, Environment and Materials" (20205012). We also thank Prof. Tomoya Imai at Kyoto University in Japan for fruitful discussion about the XRD analyses. 
Conflict of Interest

The authors declare no competing financial interest.

\section{References}

[1] M. F. Ashby, H. Shercliff, D. Cebon, Materials: engineering, science, processing and design, Butterworth-Heinemann, 2018.

[2] Z. Shan, E. A. Stach, J. M. K. Wiezorek, J. A. Knapp, D. M. Follstaedt, S. X. Mao, Science 2004, 305, 654-657.

[3] X. Qian, J. Zhou, G. Chen, Nature Materials 2021, 1.

[4] P. Y. Huang, C. S. Ruiz-Vargas, A. M. van der Zande, W. S. Whitney, M. P. Levendorf, J. W. Kevek, S. Garg, J. S. Alden, C. J. Hustedt, Y. Zhu, J. Park, P. L. McEuen, D. A. Muller, Nature 2011, 469, 389-392.

[5] P. Podsiadlo, A. K. Kaushik, E. M. Arruda, A. M. Waas, B. S. Shim, J. Xu, H. Nandivada, B. G. Pumplin, J. Lahann, A. Ramamoorthy, N. A. Kotov, Science 2007, 318, 80-83.

[6] Y. Hou, Q.-F. Guan, J. Xia, Z.-C. Ling, Z. He, Z.-M. Han, H.-B. Yang, P. Gu, Y. Zhu, S.-H. Yu, ACS nano 2020, 15, 1310-1320.

[7] B. Goris, S. Bals, W. Van den Broek, E. Carbó-Argibay, S. Gómez-Graña, L. M. Liz-Marzán, G. Van Tendeloo, Nature Materials 2012, 11, 930-935.

[8] R. J. Moon, A. Martini, J. Nairn, J. Simonsen, J. Youngblood, Chem. Soc. Rev. 2011, 40, 3941-3994.

[9] D. J. Cosgrove, Nat Rev Mol Cell Biol 2005, 6, 850-861.

[10] Y. Nishiyama, P. Langan, H. Chanzy, J Am Chem Soc 2002, 124, 9074-9082.

[11] K. Daicho, T. Saito, S. Fujisawa, A. Isogai, Acs Applied Nano Materials 2018, 1, 5774-5785.

[12] A. N. Fernandes, L. H. Thomas, C. M. Altaner, P. Callow, V. T. Forsyth, D. C. Apperley, C. J. Kennedy, M. C. Jarvis, Proc Natl Acad Sci U S A 2011, 108, E1195-1203.

[13] A. D. French, S. Pérez, V. Bulone, T. Rosenau, D. Gray, in Encyclopedia of Polymer Science and Technology, pp. 1-69.

[14] T. Saito, Y. Nishiyama, J. Putaux, M. Vignon, A. Isogai, Biomacromolecules 2006, 7, 1687-1691.

[15] T. Saito, T. Uematsu, S. Kimura, T. Enomae, A. Isogai, Soft Matter 2011, 7, 8804-8809.

[16] J. Nemoto, T. Saito, A. Isogai, ACS Appl Mater Interfaces 2015, 7, 19809-19815. 
[17] M. Zhao, F. Ansari, M. Takeuchi, M. Shimizu, T. Saito, L. Berglund, A. Isogai, Nanoscale Horizons 2018, 3, 28-34.

[18] M. Shimizu, T. Saito, A. Isogai, J. Membr. Sci. 2016, 500, 1-7.

[19] S. Huang, M. Makarem, S. N. Kiemle, Y. Zheng, X. He, D. Ye, E. W. Gomez, E. D. Gomez, D. J. Cosgrove, S. H. Kim, Carbohydrate polymers 2018, 197, 337-348.

[20] M. Shimizu, T. Saito, A. Isogai, Biomacromolecules 2014, 15, 1904-1909.

[21] Q. Zhou, E. Malm, H. Nilsson, P. T. Larsson, T. Iversen, L. A. Berglund, V. Bulone, Soft Matter 2009, 5, 4124-4130.

[22] L. Salmén, J. S. Stevanic, Cellulose 2018, 25, 6333-6344.

[23] Y. Peng, D. J. Gardner, Y. Han, A. Kiziltas, Z. Cai, M. A. Tshabalala, Cellulose 2013, 20, 2379-2392.

[24] T. Willhammar, K. Daicho, D. N. Johnstone, K. Kobayashi, Y. Liu, P. A. Midgley, L. Bergström, T. Saito, ACS nano 2021, 15, 2730-2737.

[25] Y. Ogawa, Nanoscale 2019, 11, 21767-21774.

[26] G. Nystrom, M. Arcari, J. Adamcik, I. Usov, R. Mezzenga, ACS Nano 2018, 12, 5141-5148.

[27] B. Song, S. Zhao, W. Shen, C. Collings, S.-Y. Ding, Frontiers in Plant Science 2020, 11

[28] T. Rosén, H. He, R. Wang, C. Zhan, S. Chodankar, A. Fall, C. Aulin, P. T. Larsson, T. Lindström, B. S. Hsiao, ACS Nano 2020, 14, 16743-16754.

[29] D. P. Oehme, M. S. Doblin, J. Wagner, A. Bacic, M. T. Downton, M. J. Gidley, Cellulose 2015, 22, 35013520 .

[30] See, for example, the website of Nanocellulose Japan, Available from: https://www.nanocellulosejapan.com/

\section{Figures}



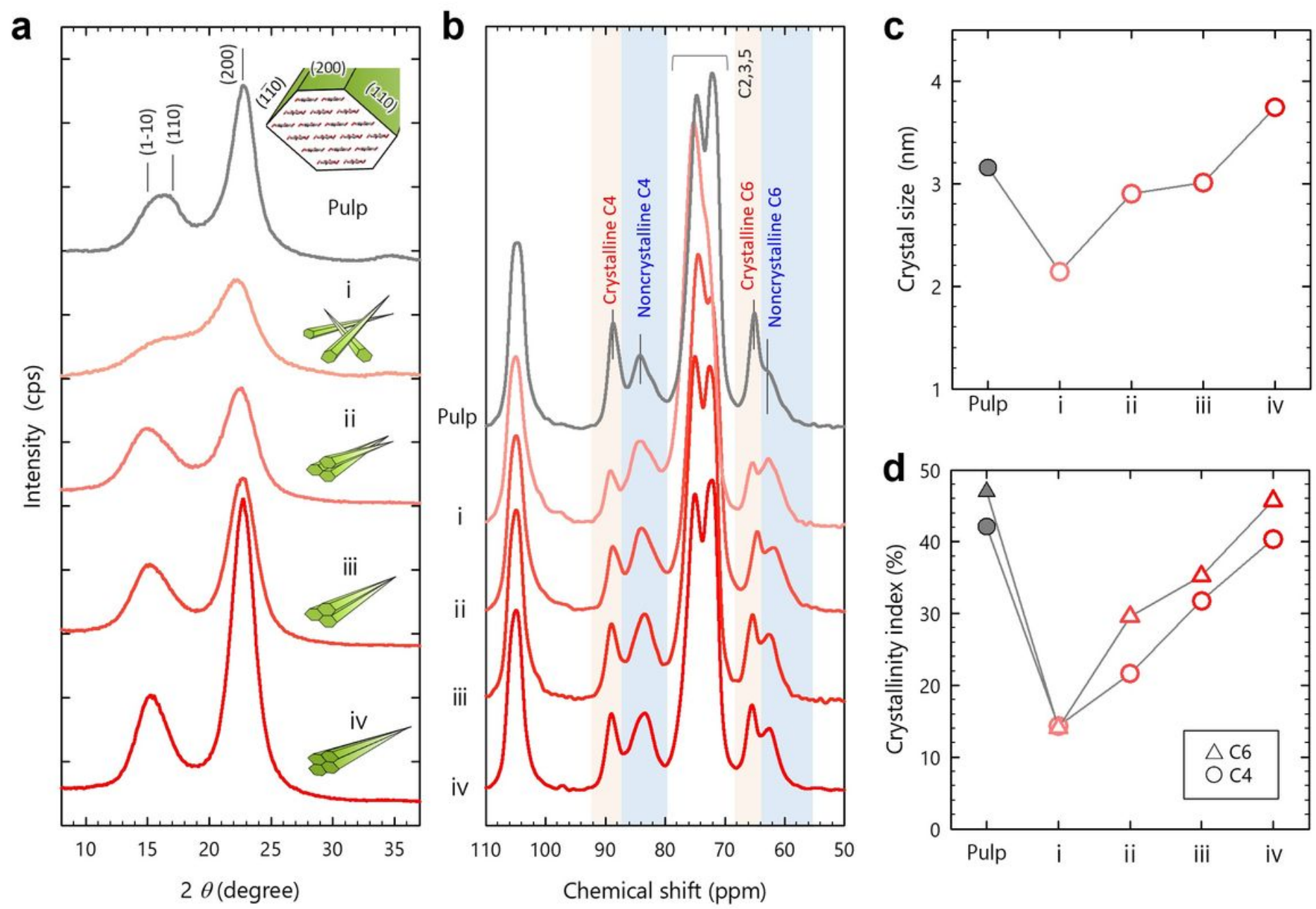

Figure 1

Recovery of crystallinity. (a) XRD profiles and (b) CP/MAS 13C NMR spectra of a series of samples ranging from the starting pulp to CNF samples i-iv. (c) Crystal size of the $(200)$ plane calculated from the XRD profiles, and (d) crystallinity indices of the $\mathrm{C} 4$ and $\mathrm{C} 6$ carbon atoms calculated from the NMR spectra.

a

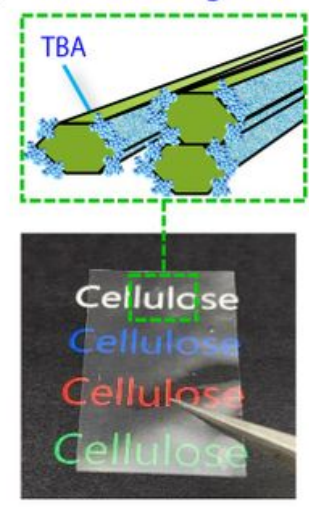

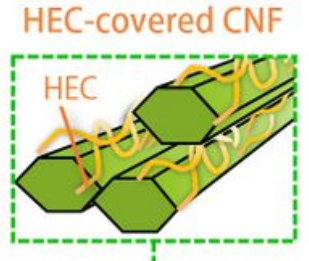

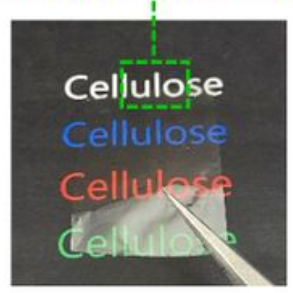

b

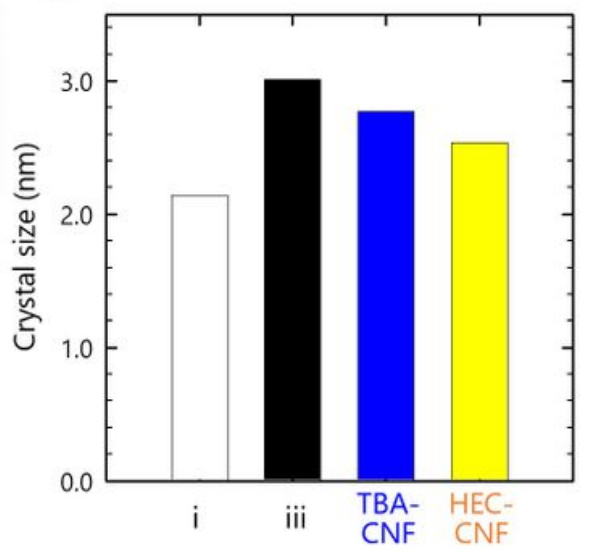

C

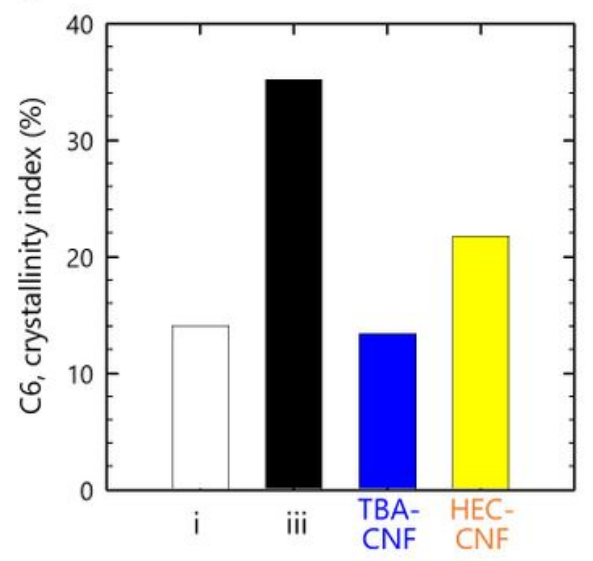

Figure 2 
Contribution of the inter-CNF interactions. (a) Schematics of the TBA-bearing and HEC-covered CNFs, and appearances of the samples. (b) Crystal sizes and (c) crystallinity indices of the $\mathrm{C} 6$ carbon atoms of samples $\mathrm{i}$ and $\mathrm{iii}$, and the TBA-bearing and HEC-covered CNFs.
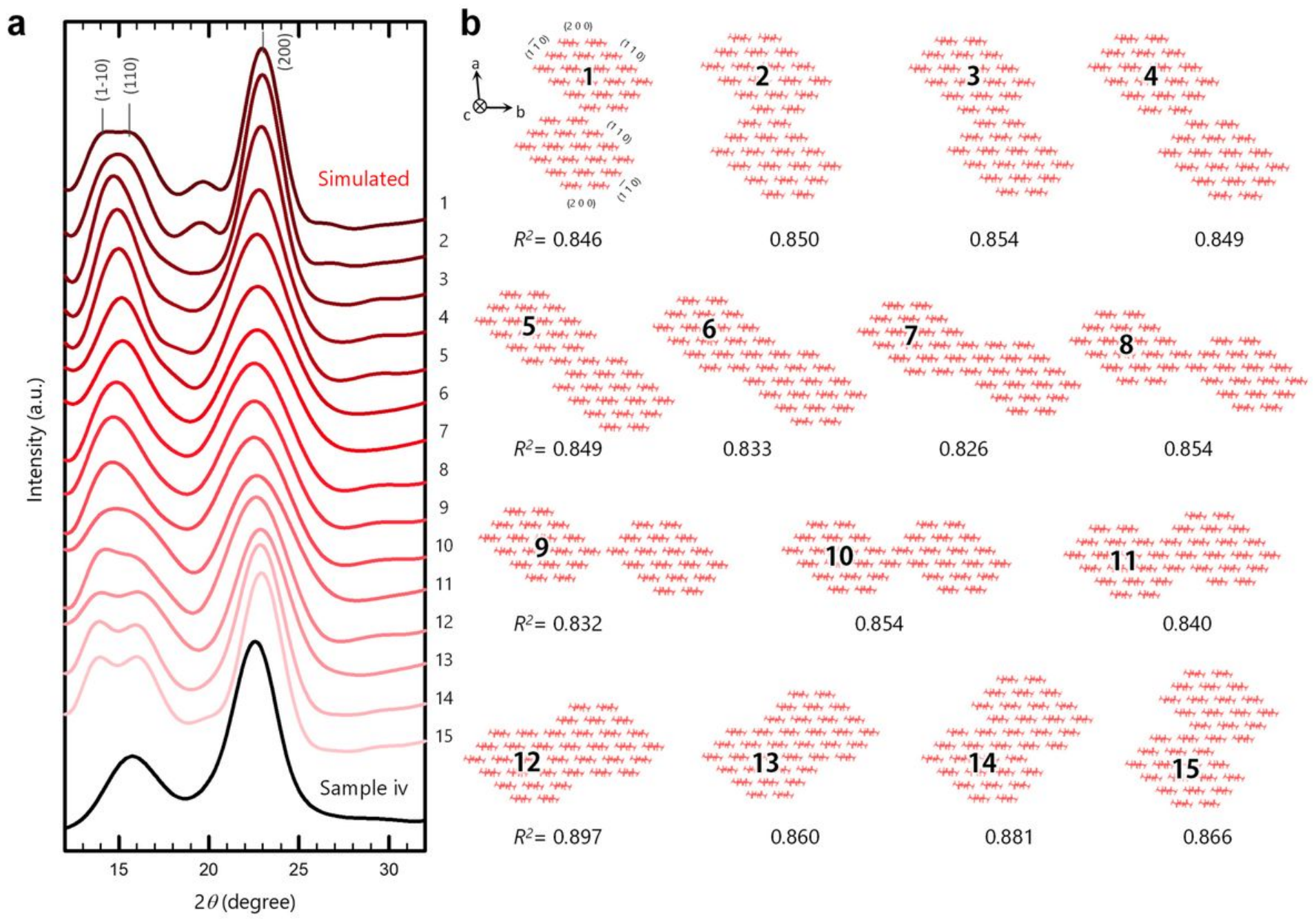

$R^{2}=0.832$

0.854

0.840
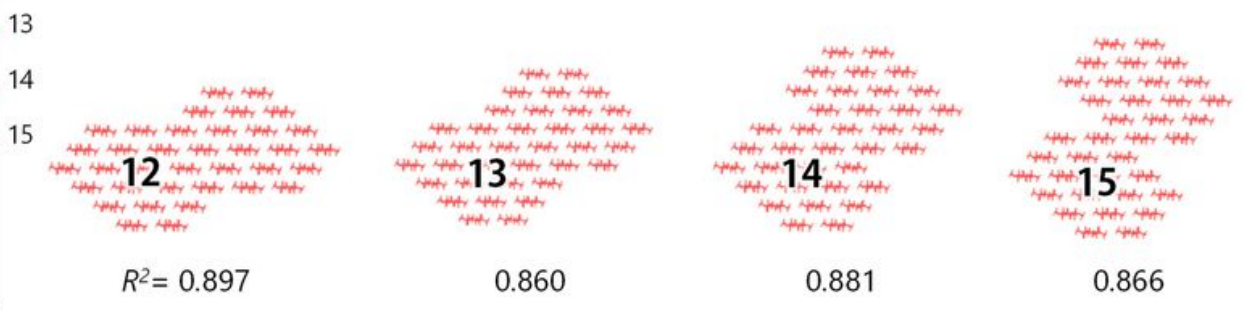

\section{Figure 3}

Simulation. Simulation. (a) Comparison of the XRD profile of sample iv with the simulated profiles of (b) the possible inter-CNF configurations \#1-15. The CNFs were assumed to assemble parallel to one another along the crystallographic c axis. 


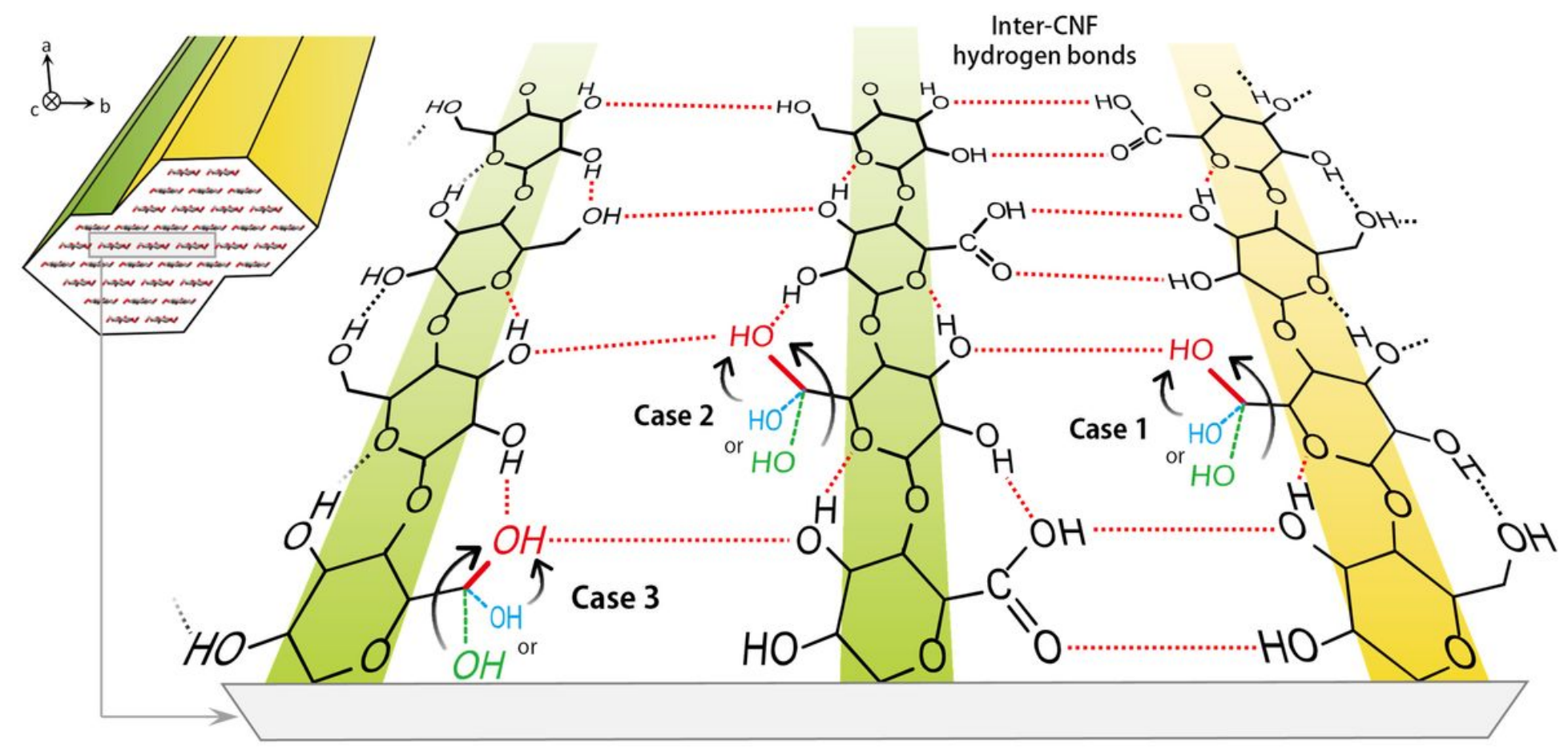

Figure 4

A model for the conformational changes of the $\mathrm{C} 6$ carbon atoms induced by the inter-CNF hydrogen bonding.

a

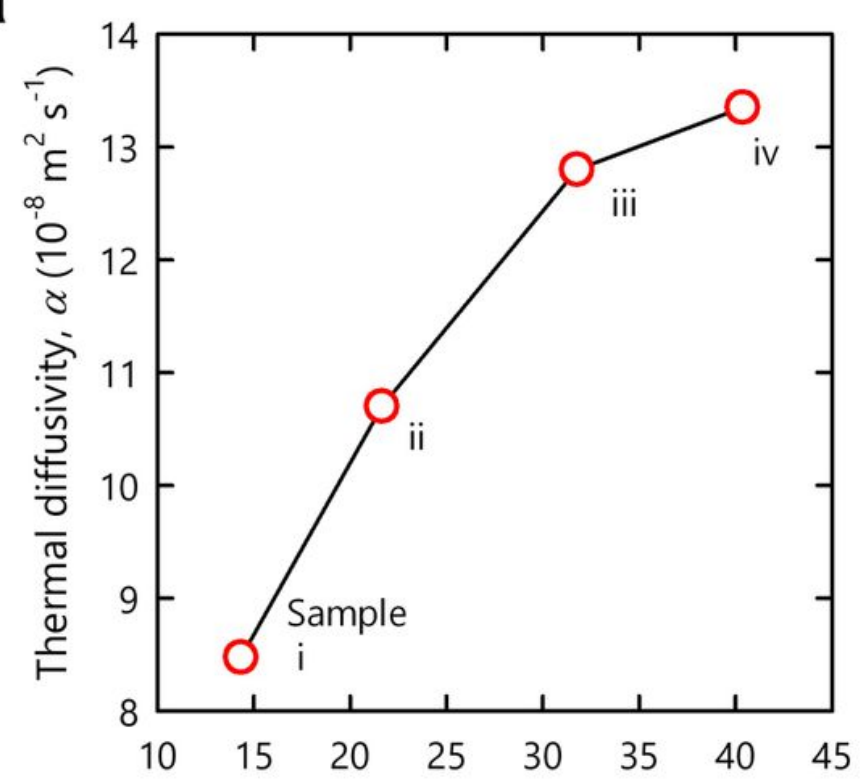

C4, crystallinity index (\%)

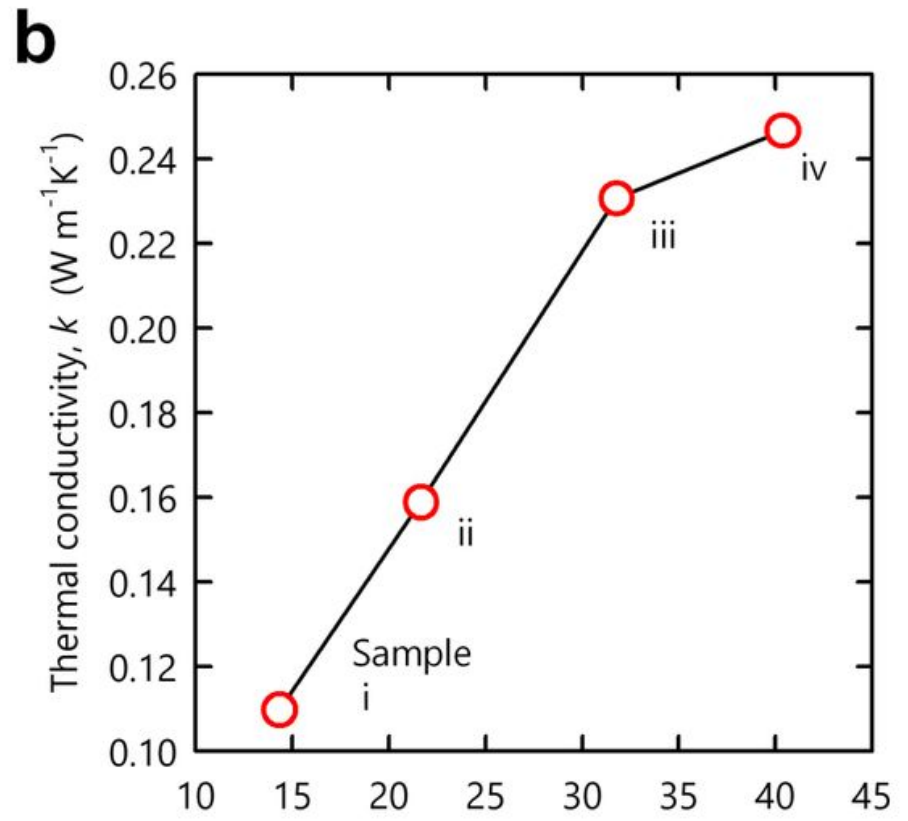

C4, crystallinity index (\%)

Figure 5

Contribution to bulk properties. (a) Thermal diffusivity and (b) conductivity of samples i-iv as a function of the crystallinity index of the $\mathrm{C} 4$ carbon atoms. 


\section{Supplementary Files}

This is a list of supplementary files associated with this preprint. Click to download.

- ACSNanoDaichoSI.pdf

- TableofContentsGraphic.pdf

- Scheme1.pdf 